\title{
Screening properties of quark-anti-quark correlators in deconfined medium
}

\author{
Swagato Mukherjee ${ }^{\dagger}$ \\ Fakultät für Physik, Universität Bielefeld, D-33615 Bielefeld, Germany. \\ E-mail: smukheraphysik.uni-bielefeld.de
}

\begin{abstract}
We present results for the inverse screening lengths (i.e. the screening masses) of quark-antiquark systems belonging to different spin-parity channels. The results are obtained from lattice QCD simulations of $2+1$ flavors of dynamical quarks with masses that correspond to realistic pion and almost physical kaon masses at zero temperature. For the light quarks our study shows that the screening masses in the pseudo-scalar and the isovector scalar channels do not become equal at the chiral crossover of QCD. This indicate that the effective restoration of the axial symmetry does not coincide with the chiral crossover of QCD. Moreover, even in the limit of zero lattice spacing the screening masses in the pseudo-scalar and the vector channels do not become degenerate at a moderately high temperature of $420 \mathrm{MeV}$. For the charm quarks we found that the screening masses in the pseudo-scalar and the vector channels remain almost equal to their zero temperature masses for temperatures less than $300 \mathrm{MeV}$. We also present a more detailed study of the screening masses in the charm quark sector by employing periodic boundary conditions along the temporal direction.
\end{abstract}

8th Conference Quark Confinement and the Hadron Spectrum

September 1-62008

Mainz, Germany

${ }^{*}$ Speaker.

$\dagger$ On behalf of the RBC-Bielefeld collaboration. The numerical calculations presented here have been carried out on the apeNEXT computers of Bielefeld University, the QCDOC computers at BNL and the BlueGene/L computer at the New York Center for Computational Sciences which is supported by the U.S. Department of Energy under Contract No. DE-AC02-98CH10886 and by the State of New York. 


\section{Introduction}

It is generally believed [1] that a thermalized deconfined medium has been created during the heavy-ion collision experiments at the Relativistic Heavy Ion Collider (RHIC) . In order to understand the structure and properties of such a deconfined medium in detail it is important to know how a system of quark-anti-quark pair behave inside such a medium. By studying the in-medium properties of different quark-anti-quark systems one can gather information, such as what are the important length-scales in the medium, what are the relevant degrees of freedom of the medium etc. Apart from these information, in-medium properties of quark-anti-quark systems help us to understand whether the chiral and the $U_{A}(1)$ axial symmetries of QCD get restored in a deconfined medium. Since the medium formed in the RHIC experiment is believed to be strongly coupled [1] a non-perturbative study of the in-medium properties of the quark-anti-quark systems are called for. To date, finite-temperature lattice QCD simulations remain the most viable and successful technique for such non-perturbative studies. However, in a finite-temperature lattice QCD simulation the maximum available physical temporal extent is always limited by the inverse of the temperature. Due to this reason, it is difficult to extract information about the energy spectrum, i.e. to extract the pole mass, of a quark-anti-quark system from its temporal correlation function. Thus, conventional finite-temperature lattice QCD simulations have long been concentrated on studying the spatial correlation functions of quark-anti-quark pairs. From the exponential decays of such spatial correlation functions one can extract the inverse of screening lengths, i.e. the screening masses [2], of quark-anti-quark systems inside a thermalized deconfined medium.

In general the pole mass $\left(m_{M}\right)$ of a quark-anti-quark system is not identical to its screening mass $\left(M^{S}\right)$. However, it is important to note that both the spatial and the temporal correlation functions depend on the same spectral density of the quark-anti-quark system [3]. From this it follows that if the quark-anti-quark system forms a stable (free) meson, e.g. at temperature $T=0$, then the screening and the pole mass become identical, i.e. $M^{S}=m_{M}$. On the other hand, if the quark-anti-quark system dissociates into free quark and anti-quark then the screening mass will be given by [4] $M_{\text {free }}^{s}(T)=2 \sqrt{(\pi T)^{2}+m_{q}^{2}}$, where $m_{q}$ is the mass of the quark. From now on the value of $M_{\text {free }}^{s}\left(T, m_{q}=0\right) / T=2 \pi T$ will be mentioned as the "Free continuum" limit. In the limit of very high temperature perturbative calculations [5] show that this "Free continuum" limit is reached from above.

Lattice computations of the screening masses of quark-anti-quark systems have a long history. A review of some of the earlier results can be found in [3]. For some of the more recent results see $[6,7]$. In the present work we have computed the screening masses of quark-anti-quark systems using lattice QCD simulations of two degenerate dynamical up $(u)$, down $(d)$ quarks and a dynamical strange $(s)$ quark. Since the explored temperatures are much smaller than the charm $(c)$ quark mass the charm quark was not treated as a dynamical flavor. The simulations were performed using an improved form of the staggered fermion action, viz. p4fat 3 . The quark masses have been chosen in a way that correspond to zero temperature meson masses $m_{\pi} \simeq 220 \mathrm{MeV}, m_{K} \simeq 500$ $\mathrm{MeV}, m_{\Psi} \simeq 3097 \mathrm{MeV}$ and $m_{\eta_{c}} \simeq 2980$. In order to have a better control over the finite lattice spacing effects we have used three different lattice cut-offs corresponding to three different values for the temporal extent $N_{\tau}=4,6$ and 8. The spatial volumes $(V)$ were kept fixed at $T V^{1 / 3}=4$. Further details of these simulations can be found in $[8,9]$. Using these gauge configurations we 


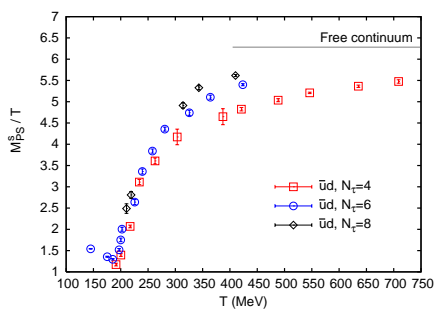

(a)

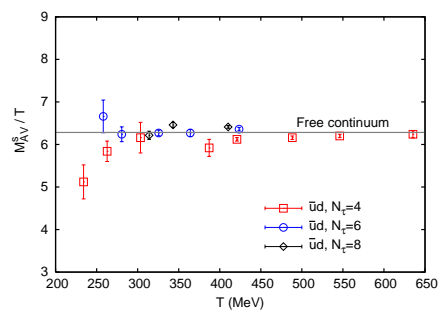

(d)

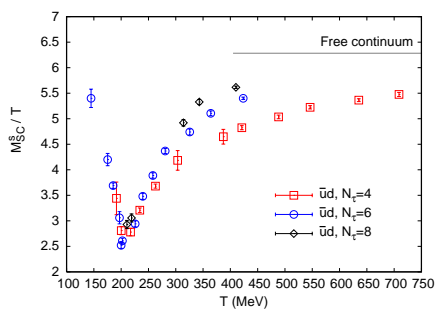

(b)

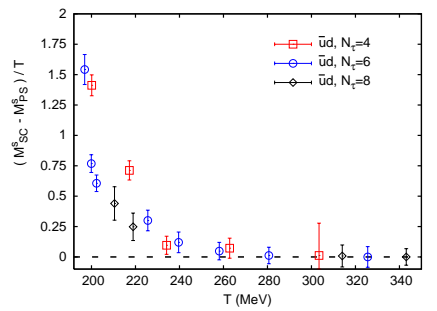

(e)

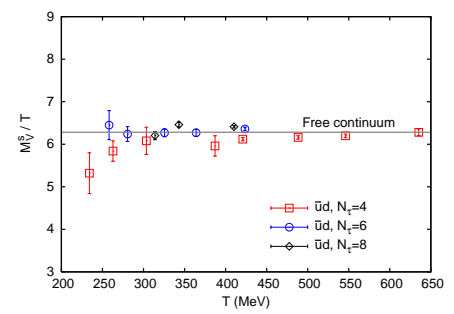

(c)

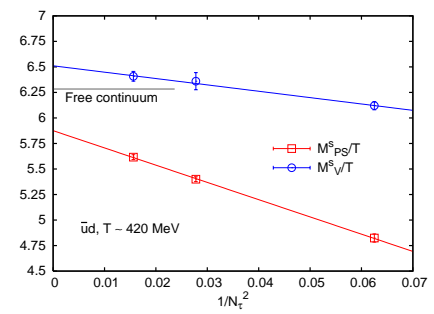

(f)

Figure 1: Screening masses in the $P S$ (a), $S C$ (b), $V$ (c) and $A V$ (d) channels for the $\bar{u} d$ sector. (e) Difference between the screening masses in the $S C$ and $P S$ channels. (f) Extrapolations of $M_{V}^{S}$ and $M_{P S}^{s}$ to zero lattice spacing at $T \simeq 420 \mathrm{MeV}$.

have analyzed the screening masses for different staggered quark-anti-quark operators [10] corresponding to the (isovector) scalar (SC), pseudo-scalar $(P S)$, (transverse) vector $(V)$ and (transverse) axial-vector $(A V)$ spin-parity channels and for four different combinations of quark fields, viz. $\bar{u} d$, $\bar{u} s, \bar{s} s$ and $\bar{c} c$.

\section{Results}

The screening masses corresponding to four different spin-parity channels are shown in Fig. 1 for the $\bar{u} d$ sector. Below the chiral crossover temperature, $T_{p c} \simeq 190 \mathrm{MeV}$, the screening masses in the $P S$ channel remain approximately equal to $m_{\pi}$. For $T>T_{p c} M_{P S}^{s}$ increases rapidly, but remains well below the "Free continuum" limit upto $T \simeq 420 \mathrm{MeV}$. Interestingly $M_{S C}^{s}$ possess a distinct minimum around $T_{p c}$, indicating that the spatial correlation length of the light (isovector) scalar quark-anti-quark pair becomes large during the chiral crossover. On the other hand, for temperatures larger than that of the chiral crossover we found that $M_{V}^{S} \simeq M_{A V}^{s} \simeq 2 \pi T$. The qualitative features of the screening masses for the $\bar{u} s$ and $\bar{s} s$ sectors were found to agree with that for the $\bar{u} d$ sector.

Once the chiral symmetry is restored the screening masses of the chiral partners are expected to be degenerate. In our case this fact is reflected in the equality of $M_{V}^{s}$ and $M_{A V}^{s}$ for $T>T_{p c}$. In contrast to this, as can be seen from Fig. 1(e), the screening masses in the (isovector) scalar and the pseudo-scalar channels do not become degenerate for temperatures $T_{p c}<T \lesssim 250 \mathrm{MeV}$. Such an observation indicates that the $U_{A}(1)$ axial symmetry does not get effectively restored at or immediately after the chiral symmetry restoration. 


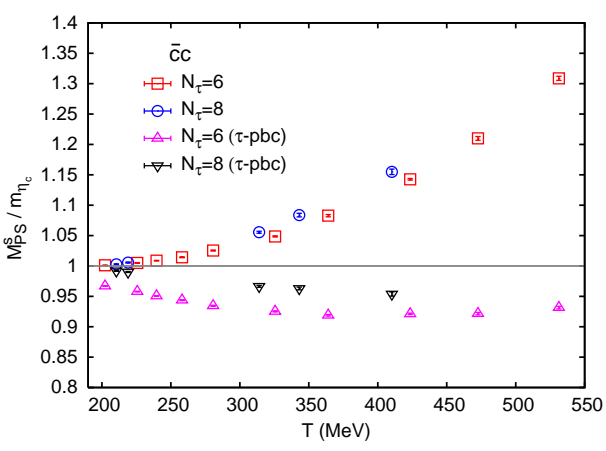

(a)

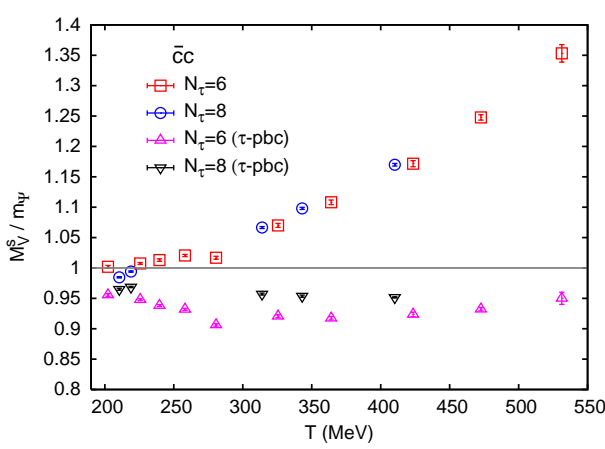

(b)

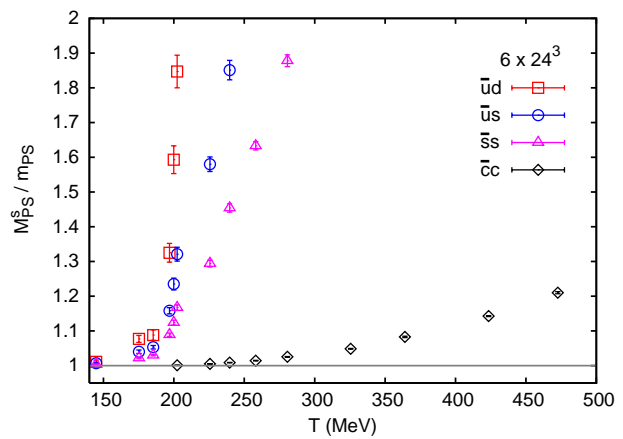

(c)

Figure 2: Ratio of the screening mass to the zero temperature pole mass in $P S$ (a) and $V$ (b) channels for the $\bar{c} c$ sector. $\tau-p b c$ means screening masses have been extracted by employing periodic boundary conditions along the temporal direction. (c) The same ratio in the $P S$ channel for four different quark-anti-quark sectors using $6 \times 24^{3}$ lattices.

In order to investigate the cut-off dependence of the screening masses more closely we have made linear extrapolations of the screening masses in lattice spacing squared $a^{2}=1 /\left(T N_{\tau}\right)^{2} \rightarrow 0$ at a temperature around $T \simeq 420 \mathrm{MeV}$ (see Fig. 1(f)). Such an extrapolation shows that $M_{P S}^{s}$ remains well below the "Free continuum" value in the limit of zero lattice spacing. On the other hand, in the limit of zero lattice spacing the screening mass in the $V$ channel becomes larger than the "Free continuum" value, in accordance with the perturbative prediction [5], even at a moderate temperature. The figure also shows that the splitting between $M_{V}^{S}$ and $M_{P S}^{S}$ persists even in the limit of zero lattice spacing. This fact is, however, in contradiction to the perturbative predictions of [5]. The overshooting of the "Free continuum" value in the $V$ channel may, however, be a finite-volume effect as observed for the lattice computations with quenched Wilson fermions [7].

In Section 1 it was discussed that if a quark-anti-quark pair forms a stable (free) mesonic state then the screening mass and the pole mass will become identical, viz. $M^{s}=m_{M}$. One can use this fact to investigate the issue of survival/dissociation of charmonia inside a deconfined medium by computing the screening mass of a $\bar{c} c$ pair and then by comparing that to the corresponding zero temperature (pole) mass. Keeping this in mind, we have analyzed the screening masses in the pseudo-scalar and the vector channels for the charm quark sector. To our surprise we found (see Fig. 2(a) and Fig. 2(b)) that the ratios $M_{P S}^{s} / m_{\eta_{c}}$ and $M_{V}^{s} / m_{\Psi}$ remain almost equal to one (within 
$<5 \%)$ till temperatures $T \lesssim 300 \mathrm{MeV}$ and then seem to show a rapid increase with increasing temperatures. The distinctiveness of the charm quark sector has been elucidated in Fig. 2(c) by comparing the ratio $M_{P S}^{S} / m_{P S}, m_{P S}$ being the (pole) mass in the $P S$ channel at $T=0$, for different flavor combinations of quark-anti-quark pairs. Notice that even for the $\bar{s} s$ sector the relative difference between the screening and the pole mass is $\sim 90 \%$ at $T \simeq 275 \mathrm{MeV}$, while for the charm quarks it is almost negligible.

In contrast to the standard anti-periodic boundary conditions one can also compute the screening masses by employing periodic boundary conditions along the temporal direction. We denote the later case by $\tau-p b c$. For the periodic boundary conditions along the temporal direction the free (continuum) screening mass will depend on the lowest bosonic Matsubara frequency $\omega_{\min }^{\text {boson }}=0$, as opposed to the lowest fermionic Matsubara frequency $\omega_{\min }^{\text {fermion }}=\pi T$ for the anti-periodic boundary conditions. Hence, $M_{\text {free } \tau-p b c}^{s}(T)=2 m_{q}$ is independent of temperature. Consequently a large difference between $M_{\text {free }}^{s}(T)$ and $M_{\text {free }, \tau-p b c}^{s}(T)$ at a fixed temperature will indicate that at that temperature the quark-anti-quark system is essentially composed of a quasi-free quark and a quasi-free anti-quark. On the other hand, if the quark-anti-quark system forms a (free) stable meson then it is expected that $M^{s}(T) \simeq M_{\tau-p b c}^{s}(T)$. Comparisons between $M^{s}(T)$ and $M_{\tau-p b c}^{s}(T)$ for $\bar{c} c$ systems are shown in Fig. 2(a) (PS channel) and Fig. 2(b) ( $V$ channel). While the difference between $M^{s}(T)$ and $M_{\tau-p b c}^{s}(T)$ remains at a level of few percent for $T \simeq 220 \mathrm{MeV}$, it increases to $\gtrsim 20 \%$ for $T \gtrsim 300 \mathrm{MeV}$. Moreover as one goes to finer lattice spacings (viz. from $a T=1 / 6$ to $a T=1 / 8$ ) the relative difference between $M^{s}(T)$ and $M_{\tau-p b c}^{s}$ reduces even further for the lower temperatures, but remains almost unchanged for $T \gtrsim 300 \mathrm{MeV}$. Thus for temperatures $T<1.5 T_{p c}$, independent of the boundary conditions along the temporal direction, the screening masses of the pseudo-scalar and the vector $\bar{c} c$ systems remain almost equal to ( $T=0$ pole) masses of $\eta_{c}$ and $J / \Psi$ mesons. Although in order to draw a firm conclusion reliable results for the spectral densities are required, based on our findings one may speculate that $\eta_{c}$ and $J / \Psi$ mesons may survive in a deconfined medium for $T<1.5 T_{p c}$.

\section{References}

[1] K. Adcox et al. [PHENIX Collaboration], Nucl. Phys. A 757, (2005) 184.

[2] C. DeTar and J. B. Kogut, Phys. Rev. Lett. 59, (1987) 399; ibid. Phys. Rev. D 36, (1987) 2828.

[3] F. Karsch and E. Laermann, in Quark Gluon Plasma III (R. Hwa ed.) [hep-lat/0305025].

[4] W. Florkowski and B. L. Friman, Z. Phys. A 347, (1994) 271.

[5] M. Laine and M. Vepsalainen, JHEP 02, (2004) 004; W. M. Alberico et al. , Nucl. Phys. A792, (2007) 152.

[6] R. V. Gavai, S. Gupta and R. Lacaze, arXiv:0803.1368 [hep-lat], I. Pushkina et al. (QCD-TARO collaboration), Phys. Lett. B 609, (2005) 265.

[7] E. Laermann et al. , PoS LAT2008, (2008) 193.

[8] M. Cheng et al., Phys. Rev. D 77, (2008) 014511.

[9] M. Cheng, PoS LAT2007, (2007) 173.

[10] S. Mukherjee, PoS LAT2007, (2007) 210. 